\title{
Watt-scale super-octave mid-infrared intrapulse difference frequency generation
}

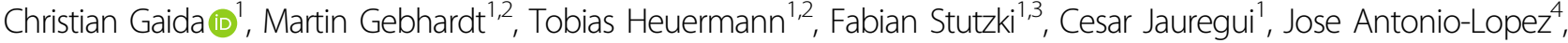 \\ Axel Schülzgen ${ }^{4}$, Rodrigo Amezcua-Correa ${ }^{4}$, Andreas Tünnermann ${ }^{1,2,3}$, loachim Pupeza ${ }^{5}$ and Jens Limpert ${ }^{1,2,3}$
}

\begin{abstract}
The development of high-power, broadband sources of coherent mid-infrared radiation is currently the subject of intense research that is driven by a substantial number of existing and continuously emerging applications in medical diagnostics, spectroscopy, microscopy, and fundamental science. One of the major, long-standing challenges in improving the performance of these applications has been the construction of compact, broadband mid-infrared radiation sources, which unify the properties of high brightness and spatial and temporal coherence. Due to the lack of such radiation sources, several emerging applications can be addressed only with infrared (IR)-beamlines in largescale synchrotron facilities, which are limited regarding user access and only partially fulfill these properties. Here, we present a table-top, broadband, coherent mid-infrared light source that provides brightness at an unprecedented level that supersedes that of synchrotrons in the wavelength range between 3.7 and $18 \mu \mathrm{m}$ by several orders of magnitude. This result is enabled by a high-power, few-cycle Tm-doped fiber laser system, which is employed as a pump at $1.9 \mu \mathrm{m}$ wavelength for intrapulse difference frequency generation (IPDFG). IPDFG intrinsically ensures the formation of carrierenvelope-phase stable pulses, which provide ideal prerequisites for state-of-the-art spectroscopy and microscopy.
\end{abstract}

\section{Introduction}

The energies of fundamental modes of atomic vibrations in molecules correspond to mid-infrared (mid-IR) frequencies in the range between several hundreds and several thousands of inverse centimeters, which are often referred to as the molecular fingerprint region (usually located in the wavenumber range between 500 and $5000 \mathrm{~cm}^{-1}$ or wavelengths between 2 and $20 \mu \mathrm{m}$ ). Molecular vibrations that exhibit electric dipole moments can be readily accessed by linear absorption spectroscopy, which causes sample-specific, spectral absorption "fingerprints" that are abundant in information about the molecular composition, structure, and conformation $^{1-5}$. For more than half a century, the powerful technique of Fourier transform IR

\footnotetext{
Correspondence: Christian Gaida (christian.gaida@uni-jena.de) ${ }^{1}$ Institute of Applied Physics, Abbe Center of Photonics, Friedrich-SchillerUniversität Jena, Albert-Einstein-Str. 15, 07745 Jena, Germany ${ }^{2}$ Helmholtz-Institute Jena, Fröbelstieg 3, 07743 Jena, Germany Full list of author information is available at the end of the article.
}

spectroscopy (FTIR) ${ }^{1}$ has enabled a plethora of applications in fields that range from analytical chemistry ${ }^{1,2}$ to environmental monitoring ${ }^{1}$ to life sciences ${ }^{3,4}$. Owing to the lack of viable alternatives and their unparalleled compactness and cost-effectiveness, thermal radiation sources are employed in the vast majority of absorption spectrometers for the mid-IR range ${ }^{1}$. However, the spatially incoherent nature of their radiation imposes a stringent trade-off between the usable IR power and the propagation distance through the sample (and through the FTIR interferometer), which causes severe limitations of the achievable signal-to-noise ratio and/or spectral resolution. This disadvantage can be overcome by IR emission of synchrotrons, which-owing to their spatial coherence-attain considerably higher brightness levels ${ }^{6}$. For FTIR measurements, this result enables higher sensitivity and the implementation of extensions, such as the combination of spectroscopy with microscopy ${ }^{4}$. However, synchrotrons are building-size facilities, which severely restricts their usability. 
During the past two decades, ultrafast laser technology has undergone rapid development that has spawned novel radiation sources and advanced spectroscopy techniques that harness their outstanding spatial and temporal coherence properties. For instance, $\mathrm{THz}$ time-domain spectroscopy ${ }^{7}$, which directly measures the complete electric field of ultrashort far-infrared pulses, has practically replaced FTIR in the region of frequencies to a few THz. Another example is precision spectroscopy, which employs frequency combs ${ }^{5}$. With appropriate detection schemes, such as the dual-comb configuration ${ }^{8}$, or FTIR with an optical delay path matched to the distance between the comb pulses ${ }^{9}$, frequency combs enable FTIRlike measurements with frequency resolutions to the comb linewidth. Applying these techniques to the previously mentioned field of applications in the mid-IR is promising for unprecedented regimes of sensitivity, spectral resolution, and acquisition time.

A laser-based source that covers the entire molecular fingerprint region and combines the key properties desirable for coherent spectroscopy does not exist: broad bandwidth (preferably without the need for tuning), spatial and temporal coherence, high repetition rate and high average power. In the absence of broadband mid-IR laser gain media, coherent broadband mid-IR sources usually employ parametric downconversion of ultrashort laser pulses generated by matured visible/near-IR laser technologies ${ }^{10-30}$. Figure 1 shows a selection of these sources, which are representative of the state of the art. These sources typically employ oxide materials as nonlinear media, which are not transparent for longer wavelengths (Fig. 1). Nonoxide crystals provide excellent transmission in the wavelength region, which ranges from 5 to $20 \mu \mathrm{m}$ (represented by light orange background color in Fig. 1); however, few crystals have low absorption at the $1 \mu \mathrm{m}$ wavelength, where most high-power ultrafast lasers operate $^{27,31}$. Among the different parametric mid-IR sources, intrapulse difference frequency generation (IPDFG) $^{24,25,27,32}$ uniquely combines compactness, intrinsic carrier-envelope phase (CEP) stability $^{33}$ and short pulse durations, which are ideal prerequisites for frequency comb spectroscopy and field-resolved spectroscopy via electro-optical sampling. However, driving lasers at $1 \mu \mathrm{m}$ exhibit two drawbacks. First, the relatively low nonlinear coefficient of LiGaS2 (LGS)-the only crystal that is suitable for multi-10 W IPDFG driven at 1 $\mu \mathrm{m}^{27,31}$ - produces a conversion efficiency of only $0.2 \%$ prior to the onset of multiphoton absorption. Second, phase matching restricts the bandwidth of the highestefficiency conversion to a few micrometers around the central wavelength.

In this study, we demonstrate that both of these limitations can be substantially alleviated with a compact source of few-cycle $1.9 \mu \mathrm{m}$ pulses and a suitable IPDFG crystal. Due to the lower photon energy of the driving pulses, nonoxide crystals with a significantly higher nonlinearity, such as GaSe, can be used ${ }^{31}$. In addition, improved phase matching conditions and broadband transmission enable the efficient generation of a broader mid-IR spectrum ${ }^{26}$. Compared with IPDFG with a $1 \mu \mathrm{m}$ driving laser ${ }^{27}$, we demonstrate an increase in conversion efficiency by one order of magnitude accompanied by a threefold increase in mid-IR bandwidth (at $-10 \mathrm{~dB}$ intensity). The table-top mid-IR source presented in this study supersedes the brightness of synchrotron radiation by several orders of magnitude over more than one octave of bandwidth while offering the benefit of intrinsic phase stability.

\section{Materials and methods}

\section{Few-cycle thulium-doped fiber laser}

A thulium-doped fiber chirped-pulse amplification system (Tm:FCPA) with an architecture that is comparable to that described in ref. ${ }^{34}$ served as the laser source for a subsequent nonlinear self-compression stage ${ }^{30}$ and intrapulse DFG. In contrast to the result in ref. ${ }^{33}$, the Tm:FCPA was operated with larger spectral bandwidth centered at $1920 \mathrm{~nm}$ and delivered at $110 \mathrm{fs}$ pulses (intensity FWHM duration). In addition, detrimental propagation effects, such as thermal blooming and pulse quality degradation from the absorption of atmospheric water vapor at wavelengths $<1930 \mathrm{~nm}$, were efficiently mitigated by placing high-power sections of the laser in vacuum ${ }^{35}$. The Tm:FCPA in this experiment delivered $31.4 \mathrm{~W}$ of average power, which corresponds to $24 \mu \mathrm{J}$ of pulse energy at a repetition rate of 1.25 $\mathrm{MHz}$. These pulses were nonlinearly compressed in an antiresonant hollow-core fiber (ARHCF) with a length of $55 \mathrm{~cm}$ and a core diameter of $51 \mu \mathrm{m}$. The input side of the ARHCF was held at 0.4 bar helium to reduce the effects of molecular water absorption while providing sufficient convection cooling of the fiber tip for long-term operation. The fiber output side was held at 3 bar argon as nonlinear medium, which causes a pressure gradient in the hollow core and significant spectral broadening of the pulses from the Tm:FCPA due to self-phase modulation upon propagation along the ARHCF. Simultaneously, the pulses were compressed due to the anomalous dispersion of the ARHCF in this wavelength region. The output of the ARHCF was characterized by measuring the intensity autocorrelation (Fig. 2a) and the nonlinearly broadened spectrum (Fig. 2b). In addition, numerical modeling of the nonlinear selfcompression stage was performed by solving the generalized nonlinear Schrödinger equation, including the ionization level of the helium gas and the transmission properties and dispersion of the $\mathrm{ARHCF}^{30}$. The numerical results match the experimental features in the temporal and spectral domain, which reveals a pulse duration of $16 \mathrm{fs}$ and a peak power of $0.9 \mathrm{GW}$ (inset of Fig. 2a). 


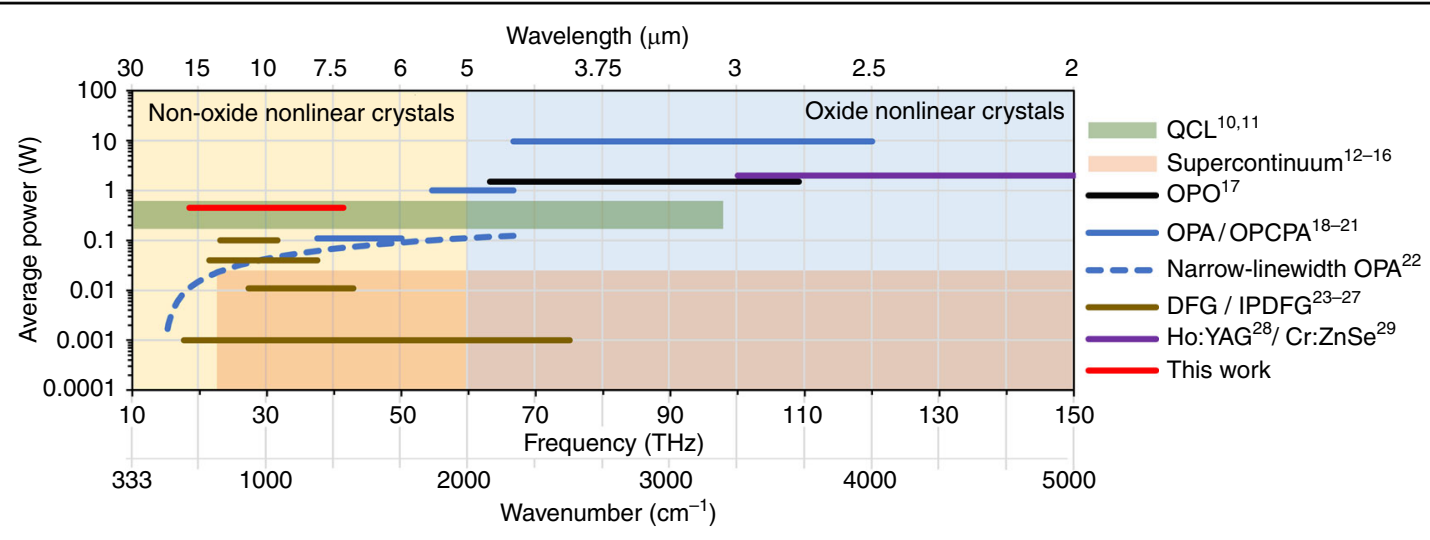

Fig. 1 State-of-the-art mid-IR sources ${ }^{10}$. Only a few concepts, such as quantum-cascade lasers ${ }^{11,}{ }^{12}\left(\mathrm{QCL}\right.$, green area) or $\mathrm{CO}_{2}$ lasers ${ }^{13}$, provide direct mid-IR generation. However, these sources emit rather narrow spectra. Supercontinuum generation (supercontinuum, orange area) in nonlinear fibers ${ }^{14-16}$, waveguides ${ }^{17}$ and crystals $^{13}$ represent extensively explored routes towards broad mid-IR spectra that are limited to average powers between $10 \mu \mathrm{W}$ and $50 \mathrm{~mW}$. Frequency downconversion in nonlinear crystals is a prominent alternative technique to obtain high power mid-IR emission. Operating in the transparency window for oxide nonlinear crystals (blue-colored background), optical parametric oscillators (OPO, black line $)^{18}$ have attained watt-level and optical parametric amplifiers (OPA, blue lines) $)^{19,20}$ and have attained 10 Watt-level average output power at a wavelength of up to $5 \mu \mathrm{m}$. OPAs and OPCPAs with longer wavelength emission have been realized by utilizing the transparency properties of nonoxide nonlinear crystals (light orange colored background), which provide an extended transparency window ${ }^{21,22}$. Steinle et al. demonstrated the advantage of a long wavelength driver in conjunction with nonoxide nonlinear crystals with a narrow-linewidth, cascaded OPA (narrow-linewidth OPA, dashed blue line) that attain average powers in the $1-100 \mathrm{~mW}$ range and tunability between 4.5 and $20 \mu \mathrm{m}^{23}$. Direct pumping at longer wavelengths for broadband difference frequency generation (DFG, brown line) or intrapulse DFG (IPDFG, brown line), however, has so far been relatively unattractive because the used pump lasers either had poor average-power scalability ${ }^{24,25}$ or could only attain high pulse energies at the expense of relatively low repetition rates in the $\mathrm{kHz}_{\text {rang }} \mathrm{e}^{26}$. Utilizing a short wavelength driver for IPDFG in a nonoxide nonlinear crystal has resulted in a $100 \mathrm{~mW}$ average power broadband frequency comb that is spectrally centered at $11.5 \mathrm{\mu m}^{27}$. In addition to the ongoing development of high average power Ho:YAG thin-disk oscillators ${ }^{28}$ and mid-IR Cr:ZnSe-bulk lasers ${ }^{29}$ (Ho:YAG / Cr:ZnS, violet line), the results presented in this work (red line) are based on the recent development of high-power Tm-doped fiber laser emitting few-cycle pulses that are centered at $1.9 \mu \mathrm{m}$ wavelength ${ }^{30}$ as a driving source for IPDFG
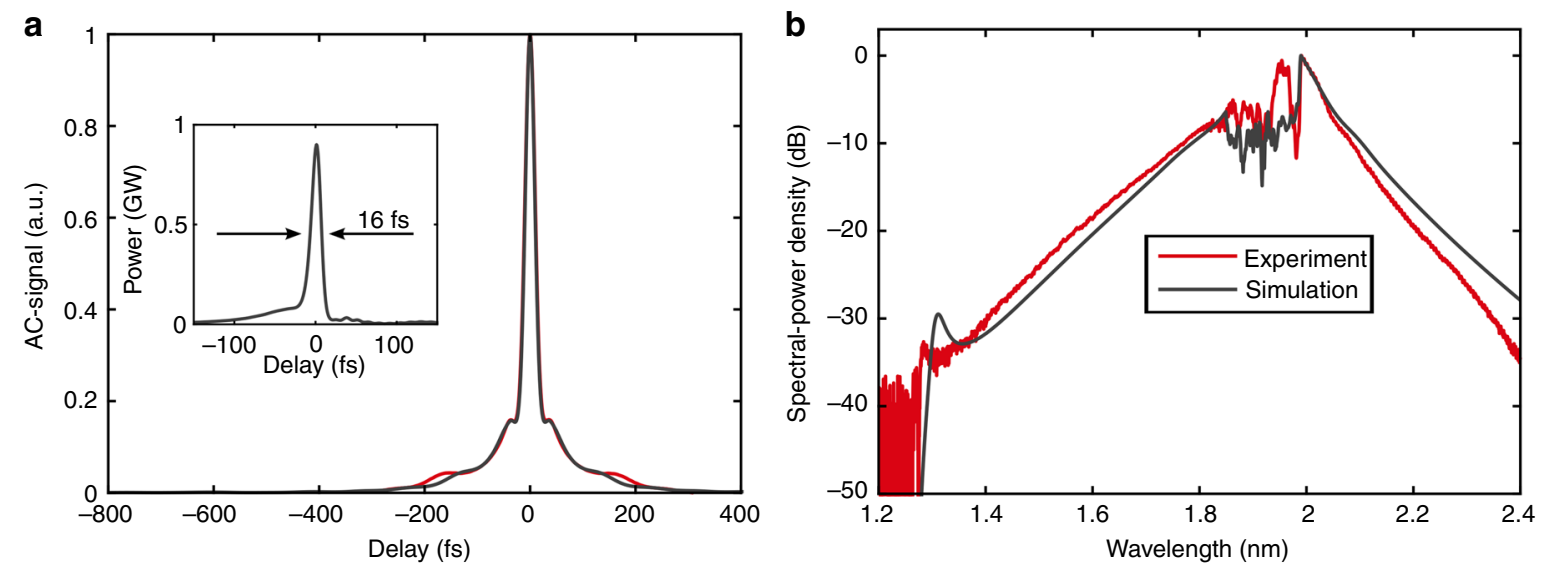

Fig. 2 Output characteristics of the few-cycle thulium-doped fiber laser for intrapulse difference frequency generation (IPDFG). a Measured (red) and simulated (black) intensity autocorrelation of the nonlinear compressed pulses. The inset shows the numerically retrieved intensity envelope of the pulse with a peak power of $0.9 \mathrm{GW}$. b Measured spectrum (red) and simulated (black) spectrum of the nonlinearly compressed pulses emitted by the Tm:FCPA-seeded antiresonant hollow core fiber at $1.25 \mathrm{MHz}$ repetition rate

\section{Intrapulse difference frequency generation in GaSe}

The IPDFG setup is schematically shown in Fig. 3a. The self-compressed pulses from the ARHCF were collimated by an $f=50 \mathrm{~mm}$ parabola that was directed to a separate chamber filled with 1 bar helium and focused on a 1-mm- thick GaSe nonlinear crystal with an $f=300 \mathrm{~mm}$ spherical mirror. This step produced a beam focus of $380 \mu \mathrm{m}$ $1 / \mathrm{e}^{2}$ width (Fig. 3b), which generated a peak intensity of $1.59 \mathrm{TW} / \mathrm{cm}^{2}$ at the input facet of the GaSe crystal, which is located immediately below the experimentally 


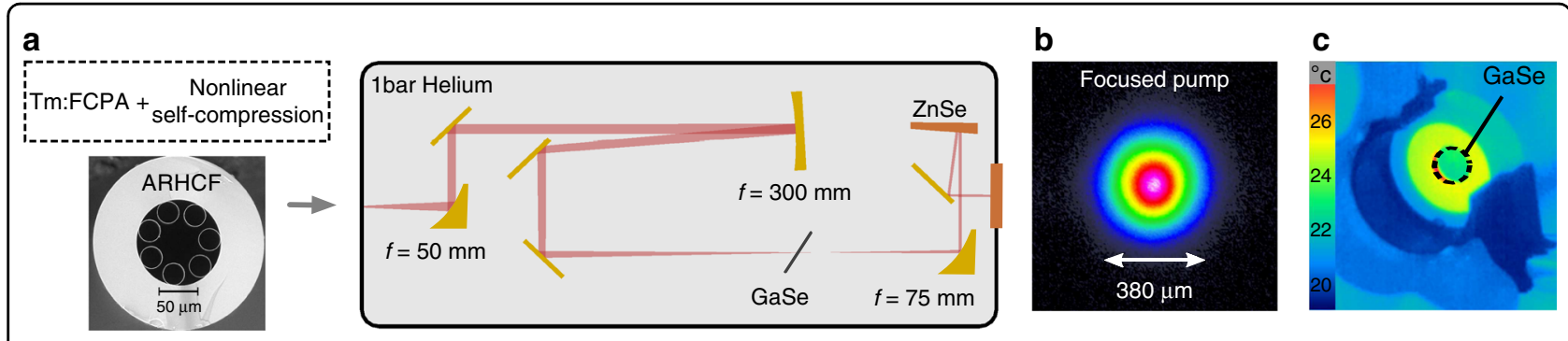

Fig. 3 Schematic experimental setup for intrapulse difference frequency generation. a The experimental setup for intrapulse DFG comprises a table-top thulium-doped fiber chirped-pulse amplification system (Tm:FCPA), an antiresonant hollow-core-fiber-based nonlinear self-compression stage and a separate chamber filled with 1 bar of helium gas for the mitigation of thermal blooming ${ }^{35}$, which enables efficient intrapulse difference frequency generation. b Beam focus with a $380 \mu \mathrm{m} 1 / \mathrm{e}^{2}$ width, which produces a peak intensity of $1.59 \mathrm{TW} / \mathrm{cm}^{2}$ at the GaSe crystal for intrapulse DFG. c Thermal camera image of the GaSe crystal, while illuminating with an $80 \mathrm{~W}$-average-power laser for several minutes

determined damage threshold of $1.7 \mathrm{TW} / \mathrm{cm}^{2}$. The generated idler and the remaining pump and signal were collimated by a $75 \mathrm{~mm}$ parabola. A ZnSe-wedge was used to reduce the total average power before the beam was directed out of the chamber. In this manner, thermal blooming due to molecular water absorption in air was efficiently mitigated, which enabled characterization of the generated idler with a Fourier transform interferometer, a thermal power meter and a mid-IR beam profiler (detection range $2-20 \mu \mathrm{m}$ ). The remaining pump light was suppressed by a longpass filter $(>40 \mathrm{~dB}$ suppression at $2 \mu \mathrm{m}$ wavelength). The losses due to the $\mathrm{ZnSe}$ wedge, $\mathrm{ZnSe}$-output window and longpass filters have been determined at a low repetition rate, which enables accurate correction of the measured average power (refer to supplementary information). The high average power handling capability of this GaSe crystal has been separately tested with a broadband amplified spontaneous emission source that provides $80 \mathrm{~W}$ of average power $(>100 \mathrm{~nm}$ width at $-10 \mathrm{~dB}$ centered at $1900 \mathrm{~nm})$. An absorption-induced thermal gradient has not been observed (Fig. 3c), which corroborates the power-scaling potential of IPDFG in GaSe.

\section{Phase matching considerations}

Efficient mid-IR generation via IPDFG requires phase matching of the pump, signal and idler waves, which can be achieved by exploiting the crystal birefringence, whereas the synchronization of the signal and pump is intrinsically ensured for transform-limited input pulses. The dispersion relations of the ordinary and extraordinary GaSe crystal axes were estimated with Sellmeier coefficients provided by the crystal manufacturer (refer to supplementary information). Based on the spectral band of the few-cycle pulses launched at the crystal, the phase mismatch $\left(\Delta k=k_{\mathrm{p}}-k_{\mathrm{s}}-k_{\mathrm{i}}\right)$ can be calculated for the pump $\left(k_{\mathrm{p}}\right)$, signal $\left(k_{\mathrm{s}}\right)$, and idler $\left(k_{\mathrm{i}}\right)$ wave vectors. Since GaSe is only available in a Z-cut orientation, the condition $\Delta k=0$ and the targeted idler wavelengths in the molecular fingerprint region can theoretically be fulfilled in three different configurations, which are schematically illustrated in Fig. 4. Simultaneous propagation of signal and pump waves in the projected extraordinary axis (e) and idler waves in the ordinary axis (eeo, phase matching type $2 \mathrm{~b}$ ) implies large external angles of incidence $(\theta)$, and consequently, large idler output angles, which cause total internal reflection of the generated mid-IR at the output side of the GaSe crystal. Phase matching and efficient mid-IR idler output can only be achieved with propagation of the pump waves in the projected $e$-axis, the signal waves in the $o$-axis and the idler waves in the $e$-or $o$-axis (type 2a, eoe or type 1, eoo). The two possible branches for phase matching and sufficiently small external angles of incidence $(\theta)$ vs. idler frequency are illustrated in Fig. $5 \mathrm{a}$. The width of each branch is attributed to sweeping the available pump wavelength from 1300 to $2300 \mathrm{~nm}$ using the remaining spectrum to the $2400 \mathrm{~nm}$ wavelength as a signal and fulfilling the condition $\Delta k=0$.

\section{Results and discussion \\ Broadband tuning in the mid-IR}

For type I phase matching (eoe) the crystal orientation and input polarization were optimized to follow the scheme shown in Fig. 4a. We tuned the crystal between $\theta$ $=29^{\circ}$ and $\theta=49^{\circ}$ external angle of incidence (Fig. 5b) to optimize for efficient broadband mid-IR generation in the $3-20 \mu \mathrm{m}$ wavelength range. This optimization was performed with a reduced pulse repetition rate of $400 \mathrm{kHz}$ to maintain the average power at a level that was sufficiently low for operation in normal atmosphere, which enabled manual tuning of the GaSe crystal. For each external angle of incidence $(\theta)$, the idler average power was maximized by slightly tuning the orientation of the crystal $\left(\Delta \phi<1^{\circ}\right)$ with respect to the input polarization. The generated idler was spectrally characterized with a Fourier transform interferometer that was equipped with a thermal sensor. The center frequency of the generated mid-IR light decreased for lower angles of incidence $(\theta)$ and fits the 

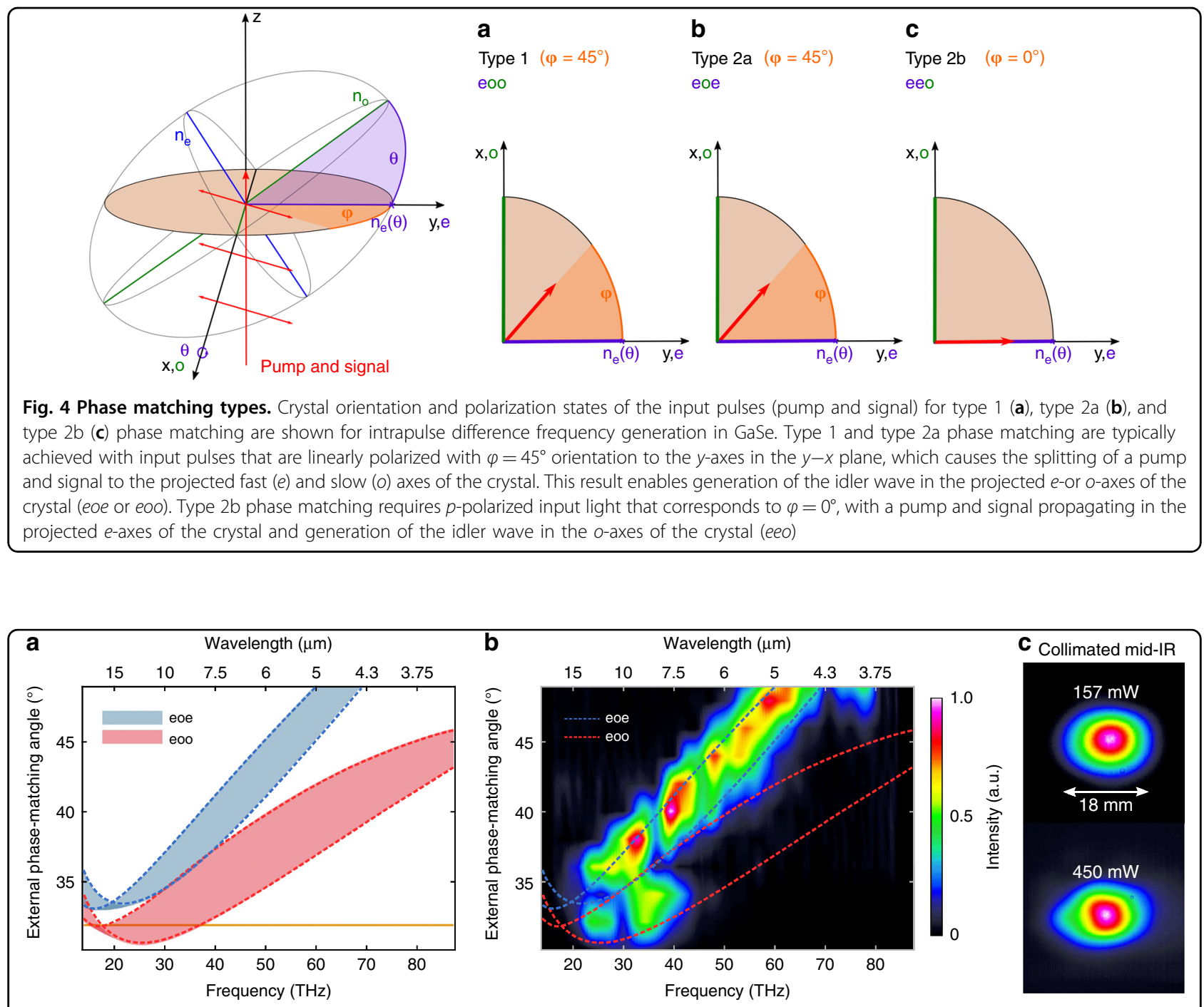

Fig. 5 Phase- matching calculations and experiment. a The phase matching calculation produces two possible branches with respect to the external angle of incidence on the GaSe-crystal and generated idler frequencies of ordinary (type 1, eoo) or extraordinary (type 2a, eoe) polarization. The width of each branch represents phase matched scenarios $(\Delta k=0)$ for intrapulse difference frequency generation (IPDFG), which considers pump waves that range from 1300 to $2300 \mathrm{~nm}$ (always e-polarized) and signal waves of up to $2400 \mathrm{~nm}$ (always o-polarized) of the few-cycle input pulse (see spectrum in Fig. 2b). The orange line indicates the external angle of incidence that was chosen for the high-power experiment. $\mathbf{b}$ Generated mid-IR spectra for a sweep of the external angle of incidence on the GaSe crystal. The blue dashed lines and red dashed lines represent the calculations for eoe phase matching and eoo phase matching, respectively. The orange line indicates the external angle of incidence that was chosen for the high-power experiment presented in this paper. c Typical collimated mid-IR beam profiles at a $400 \mathrm{kHz}$ pulse repetition rate/157 mW average power and a $1.25 \mathrm{MHz}$ pulse repetition rate/450 $\mathrm{mW}$ average power. The weak beam deformation at the highest power is caused by thermal deformation of the collimating gold parabola

theoretically predicted eoe-branch (compare with Fig. 5a). The generated mid-IR average power at a pulse repetition rate of $400 \mathrm{kHz}$ was $157 \mathrm{~mW}$ at an external input angle of $32^{\circ}$, as indicated by the orange lines in Fig. 5a, b; it did not significantly change for the entire tuning range. The beam profile at this average power level is depicted in Fig. 5c and shows no sign of beam quality degradation. The slight ellipticity can be attributed to spatial walk-off between idler and pump waves and/or small alignment errors of the collimating parabola. Angles of incidence larger than $49^{\circ}$ were not possible due to beam clipping at the crystal holder, which prevented us from observing the highfrequency limit that was ultimately set by the input spectral bandwidth. Input angles smaller than $30^{\circ}$ produced an abrupt reduction of the mid-IR average power, which indicates that no phase matching was achievable beyond this point. This observation is also consistent with the calculated phase matching conditions (Fig. 5a). The eoe-branch appears to be more efficient over a wide range of external angles of incidence. Note that experimental 
generation of idler frequencies that fit the eoo-branch for external angles smaller than $40^{\circ}$ was possible by detuning the angle $\phi$ and changing the ratio of the pump and signal intensities. However, the average mid-IR output power was significantly reduced. When optimizing for the highest mid-IR average power, the eoe-branch was always more efficient for external angles of incidence larger than $35^{\circ}$. The eoo-branch becomes similarly efficient for external angles of incidence smaller than $34^{\circ}$. This behavior cannot be understood by looking at the phase matching conditions in Fig. 5a. The significantly higher efficiency of the eoe-branch compared with the eoo-branch is caused by a complex interplay of groupdelay-dispersion mismatch and temporal chirp of the fewcycle input pulse during propagation in the extraordinary and ordinary crystal axes. This result produces a longer nonlinear interaction length of pump and signal waves that fulfill the eoe-phase matching conditions (Fig. 5b), which render the conversion process efficient, even for short idler wavelengths. The influences of group delay mismatch and temporal chirp on conversion efficiency are significantly reduced for pump and signal waves that are in close wavelength proximity, which renders generation of long idler wavelengths generally more efficient even in the case of eoo-phase matching.

\section{High brightness mid-IR generation}

The external angle of incidence at $32^{\circ}$ (refer to orange lines in Fig. 5a, b) was employed for experiments at higher average power. To avoid detrimental water vapor absorption effects in the atmosphere ${ }^{35}$ we evacuated the DFG chamber and subsequently flooded it with 1 bar of dry helium gas. The repetition rate of the few-cycle Tmdoped fiber laser was incrementally increased, which produced a proportional increase of the mid-IR output average power. At a maximum pump power of $31.4 \mathrm{~W}$, which emerges from the ARHCF, we measured an average mid-IR power of $450 \mathrm{~mW}$ with a spectrum that spans from $7.3 \mu \mathrm{m}$ to $16.5 \mu \mathrm{m}(-10 \mathrm{~dB}$ intensity bandwidth) at a pulse repetition rate of $1.25 \mathrm{MHz}$. This result corresponds to a conversion efficiency of $1.8 \%$ assuming $25 \mathrm{~W}$ of average pump power in the GaSe crystal, which accounts for Fresnel reflection at the crystal surface and losses of steering optics after the ARHCF. The collimated mid-IR beam profile is depicted in Fig. $5 \mathrm{c}$ and reveals a slight beam deformation. We attribute this to the onset of thermal degradation of the collimating gold-coated parabola after the GaSe crystal, which prevented further increase of the pulse repetition rate. Assuming a nearly diffraction-limited beam quality, the brightness of this high-power mid-IR source has been calculated (refer to supplementary information) and compared with state-ofthe-art results based on IPDFG pumped at a $1 \mu \mathrm{m}$ wavelength. The result of IPDFG pumped at a $2 \mu \mathrm{m}$ wavelength is depicted in Fig. 6 (PSD is provided in the supplementary information) and reveals a significant increase of brightness and bandwidth compared with IPDFG pumped at a $1 \mu \mathrm{m}$ wavelength ${ }^{27}$, whereas the conversion efficiency of $1.8 \%$ is higher by nearly one order of magnitude. For comparison, the brightness of thirdgeneration synchrotron radiation in this wavelength range $^{6}$, which is also depicted in Fig. 6, is lower by several orders of magnitude, which substantiates the potential of this novel table-top mid-IR source for future applications. The presented front end based on a Tm:FCPA delivers a variable pulse train that is tunable in the pulse repetition rate, which closes the gap between conventional laser systems that are capable of delivering either low repetition

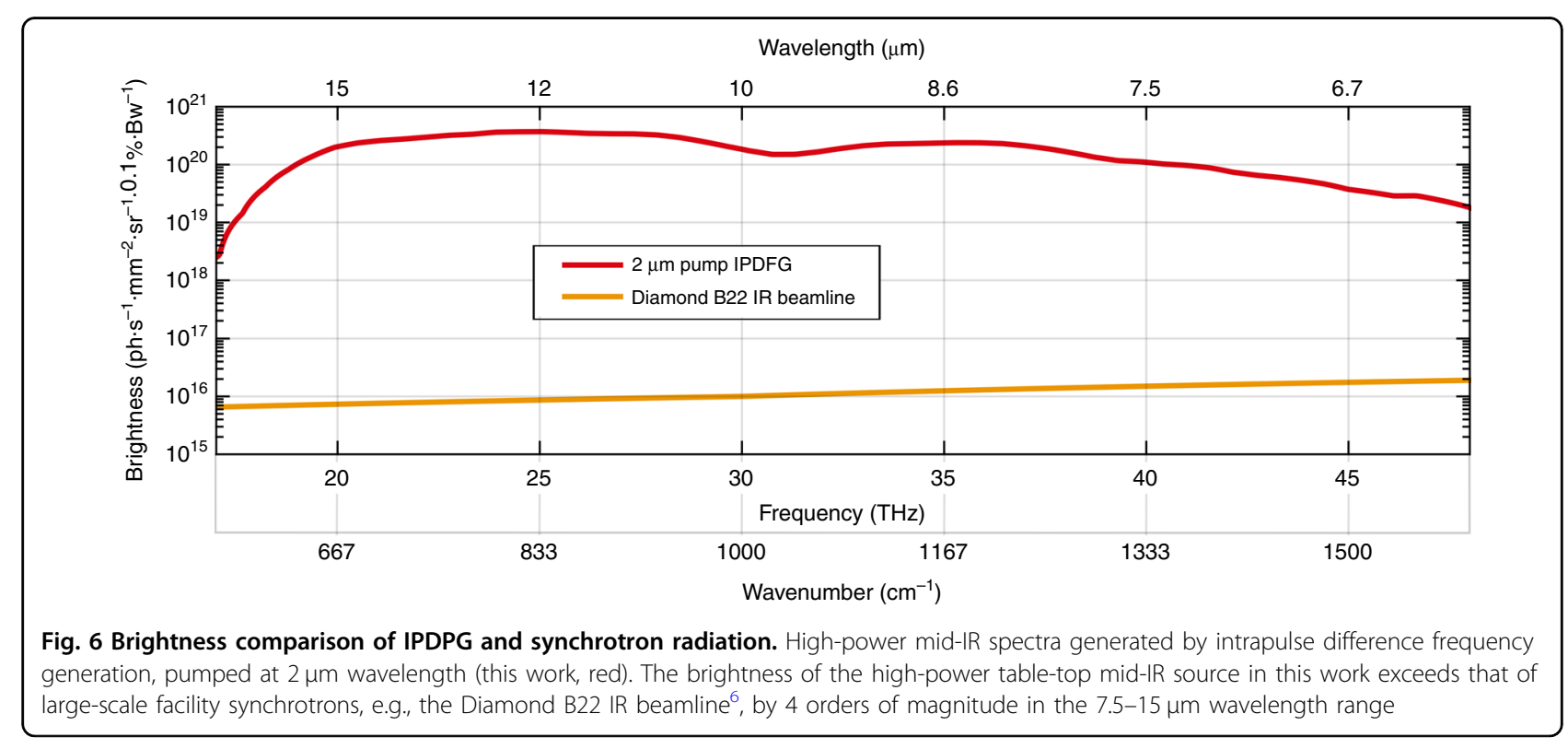


rates in the range $100 \mathrm{~Hz}-1 \mathrm{kHz}^{21,22,26}$ (low average power) or very high repetition rates in the range 10-100 $\mathrm{MHz}^{19,27,28}$ (low peak power). Thus, the system presented in this paper provides unique output parameters, which facilitates unprecedented sensitivity for background-free detection (e.g., electro-optical sampling ${ }^{27}$ ). This technique is based on nonlinear back conversion, which benefits from high peak power in the mid-IR and, in parallel, from a high photon flux.

\section{Conclusions}

We have demonstrated a source of coherent mid-IR radiation with an unprecedented combination of average power, peak power, and spectral coverage in the molecular fingerprint region. At average power in excess of $150 \mathrm{~mW}$, the spectral coverage can be tuned between 3.7 and $18 \mu \mathrm{m}$. The source is based on IPDFG, which is a nonlinear frequency downconversion process that affords the advantages of phase stability and simplicity ${ }^{24-27}$. This recent advancement in table-top Tm-doped fiber laser technology $y^{30,34,36,37}$ offers a flexible selection of laser parameters with respect to the targeted application in terms of pulse repetition rate and pulse energy $(0.5 \mathrm{~mJ} / 10$ $\mathrm{kHz}$ to $0.5 \mu \mathrm{J} / 100 \mathrm{MHz}$ ) as a starting point for mid-IR generation. The combination of GaSe as a nonlinear medium and $2-\mu \mathrm{m}$ radiation for driving IPDFG, combined with ongoing further development of ultrafast $2-\mu \mathrm{m}$ technology promises further power scalability of the scheme presented in this paper. The demonstrated power level renders this approach a truly viable table-top alternative to broadband synchrotron mid-IR radiation. In addition, IPDFG intrinsically ensures the formation of a CEP stable output pulse train $^{33}$, which provides ideal prerequisites for spectroscopic and microscopic techniques based on the control and observation of the electric field of the mid-IR radiation ${ }^{27}$.

\section{Acknowledgements}

The research that produced these results has been partly supported by the German Federal Ministry of Education and Research (BMBF) under contract "NUKLEUS" (13N13973), the United States AFOSR (FA9550-15-10041) and the United States ARO (W911NF-12-1-0450 and W911NF-17-1-0501). We thank Dr. Oleg Pronin (MPQ Garching) for help with the spectral characterization and Marinus Huber (MPQ Garching) for fruitful discussions about possible fields of applications. Martin Gebhardt and Tobias Heuermann acknowledge support by the Helmholtz-Institute Jena. Fabian Stutzki and Christian Gaida acknowledge support by the Carl Zeiss Stiftung.
}

\footnotetext{
Author details

${ }^{1}$ Institute of Applied Physics, Abbe Center of Photonics, Friedrich-SchillerUniversität Jena, Albert-Einstein-Str. 15, 07745 Jena, Germany. ${ }^{2}$ HelmholtzInstitute Jena, Fröbelstieg 3, 07743 Jena, Germany. ${ }^{3}$ Fraunhofer Institute for Applied Optics and Precision Engineering, Albert-Einstein-Str. 7, 07745 Jena, Germany. ${ }^{4}$ CREOL, College of Optics and Photonics, University of Central Florida, Orlando, FL 32816, USA. ${ }^{5}$ Max-Planck-Institute of Quantum Optics, Hans-Kopfermann-Str. 1, 85748 Garching, Germany
}

\section{Authors' contributions}

C.G., M.G., F.S., C.J., A.T., and J.L. designed and built the ultrafast Tm:fiber laser system. J.A.-L., A.S. and R.A.-C. designed and drew the antiresonant hollow-core fiber. M.G., C.G., F.S., and J.L. designed and realized the nonlinear selfcompression stage. C.G., M.G., T.H., J.L., and I.P. performed the nonlinear intrapulse difference frequency generation experiment. All authors contributed to the final manuscript.

\section{Conflict of interest}

The authors declare that they have no conflict of interest.

Supplementary information is available for this paper at https://doi.org/ 10.1038/s41377-018-0099-5.

Received: 31 August 2018 Revised: 29 October 2018 Accepted: 6 November 2018

Published online: 28 November 2018

\section{References}

1. Griffiths, P. R. \& de Haseth, J. A. Fourier Transform Infrared Spectrometry 2nd edn (Wiley, Hoboken, NJ, 2007)

2. Haas, J. \& Mizaikoff, B. Advances in mid-infrared spectroscopy for chemical analysis. Annu. Rev. Anal. Chem. 9, 45-68 (2016).

3. Baker, M. J. et al. Using Fourier transform IR spectroscopy to analyze biological materials. Nat. Protoc. 9, 1771-1791 (2014).

4. Doherty, J., Cinque, G. \& Gardner, P. Single-cell analysis using Fourier transform infrared microspectroscopy. Appl. Spectrosc. Rev. 52, 560-587 (2017).

5. Schliesser, A., Picqué, N. \& Hänsch, T. W. Mid-infrared frequency combs. Nat. Photonics 6, 440-449 (2012).

6. Cinque, G., Frogley, M. D. \& Bartolini, R. Far-IR/THz spectral characterization of the coherent synchrotron radiation emission at diamond IR beamline B22. Rend. Lince-. 22, 33-47 (2011).

7. Tonouchi, M. Cutting-edge terahertz technology. Nat. Photonics 1, 97-105 (2007).

8. Keilmann, F., Gohle, C. \& Holzwarth, R. Time-domain mid-infrared frequencycomb spectrometer. Opt. Lett. 29, 1542-1544 (2004).

9. Maslowski, P. et al. Surpassing the path-limited resolution of Fourier-transform spectrometry with frequency combs. Phys. Rev. A 93, 021802 (2016). (R).

10. Biegert, J., Bates, P. K. \& Chalus, O. New mid-infrared light sources. IEEE J. Sel. Top. Quantum Electron. 18, 531-540 (2012).

11. Rauter, P. \& Capasso, F. Multi-wavelength quantum cascade laser arrays. Laser Photonics Rev. 9, 452-477 (2015).

12. Kole, M. R., Reddy, R. K., Schulmerich, M. V., Gelber, M. K. \& Bhargava, R. Discrete frequency infrared microspectroscopy and imaging with a tunable quantum cascade laser. Anal. Chem. 84, 10366-10372 (2012).

13. Pigeon, J. J., Tochitsky, S. Y., Gong, C. \& Joshi, C. Supercontinuum generation from 2 to $20 \mu \mathrm{m}$ in $\mathrm{GaAs}$ pumped by picosecond $\mathrm{CO}_{2}$ laser pulses. Opt. Lett. 39, 3246-3249 (2014).

14. Petersen, C. R. et al. Mid-infrared supercontinuum covering the 1.4-13.3 $\mu \mathrm{m}$ molecular fingerprint region using ultra-high NA chalcogenide step-index fibre. Nat. Photonics 8, 830-834 (2014).

15. Møller, U. et al. Multi-milliwatt mid-infrared supercontinuum generation in a suspended core chalcogenide fiber. Opt. Express 23, 3282-3291 (2015).

16. Kubat, I. et al. Thulium pumped mid-infrared 0.9-9 $\mu \mathrm{m}$ supercontinuum generation in concatenated fluoride and chalcogenide glass fibers. Opt. Express 22, 3959-3967 (2014)

17. $\mathrm{Yu}, \mathrm{Y}$. et al. A broadband, quasi-continuous, mid-infrared supercontinuum generated in a chalcogenide glass waveguide. Laser Photonics Rev. 8, 792-798 (2014).

18. Adler, F. et al. Phase-stabilized, 1.5W frequency comb at 2.8-4.8 $\mu \mathrm{m}$. Opt. Lett. 34, 1330-1332 (2009).

19. Seidel, M. et al. Multi-watt, multi-octave, mid-infrared femtosecond source Science Advances 4. 1526-1534 (2018).

20. Elu, U. et al. High average power and single-cycle pulses from a mid-IR optical parametric chirped pulse amplifier. Optica 4, 1024-1029 (2017).

21. von Grafenstein, L. et al. $5 \mu \mathrm{m}$ few-cycle pulses with multi-gigawatt peak power at a 1 kHz repetition rate. Opt. Lett. 42, 3796-3799 (2017).

22. Sanchez, D. et al. $7 \mu \mathrm{m}$, ultrafast, sub-millijoule-level mid-infrared optical parametric chirped pulse amplifier pumped at 2 um. Optica 3, 147-150 (2016). 
23. Steinle, T., Mörz, F., Steinmann, A. \& Giessen, H. Ultra-stable high average power femtosecond laser system tunable from 1.33 to $20 \mu \mathrm{m}$. Opt. Lett. $\mathbf{4 1}$ 4863-4866 (2016).

24. Gambetta, A. et al. Milliwatt-level frequency combs in the 8-14 $\mu \mathrm{m}$ range via difference frequency generation from an Er: fiber oscillator. Opt. Lett. 38, 1155-1157 (2013)

25. Keilmann, F. \& Amarie, S. Mid-infrared frequency comb spanning an octave based on an Er fiber laser and difference-frequency generation. J. Infrared Millim.Terahertz Waves 33, 479-484 (2012).

26. Junginger, F. et al. Single-cycle multiterahertz transients with peak fields above 10 MV/cm. Opt. Lett. 35, 2645-2647 (2010).

27. Pupeza, I. et al. High-power sub-two-cycle mid-infrared pulses at $100 \mathrm{MHz}$ repetition rate. Nat. Photonics 9, 721-724 (2015).

28. Zhang, J. et al. Multi-mW, few-cycle mid-infrared continuum spanning from 500 to $2250 \mathrm{~cm}^{-1}$. Light Sci. Appl. 7, 17180 (2018).

29. Vasilyev, S. et al. Ultrafast middle-IR lasers and amplifiers based on polycrystalline Cr:ZnS and Cr:ZnSe. Opt. Mater. Express 7, 2636-2650 (2017).

30. Gebhardt, M. et al. Nonlinear pulse compression to $43 \mathrm{~W}$ GW-class few-cycle pulses at $2 \mu \mathrm{m}$ wavelength. Opt. Lett. 42, 4179-4182 (2017).
31. Petrov, V. Frequency down-conversion of solid-state laser sources to the midinfrared spectral range using non-oxide nonlinear crystals. Prog. Quantum Electron. 42, 1-106 (2015).

32. Fattahi, H., Schwarz, A., Keiber, S. \& Karpowicz, N. Efficient, octave-spanning difference-frequency generation using few-cycle pulses in simple collinear geometry. Opt. Lett. 38, 4216-4219 (2013).

33. Baltuška, A., Fuji, T. \& Kobayashi, T. Controlling the carrier-envelope phase of ultrashort light pulses with optical parametric amplifiers. Phys. Rev. Lett. 88, 133901 (2002).

34. Gaida, C. et al. Thulium-doped fiber chirped-pulse amplification system with 2 GW of peak power. Opt. Lett. 41, 4130-4133 (2016).

35. Gebhardt, M. et al. Impact of atmospheric molecular absorption on the temporal and spatial evolution of ultra-short optical pulses. Opt. Express 23, 13776-13787 (2015).

36. Gaida, C. et al. Self-compression in a solid fiber to $24 \mathrm{MW}$ peak power with few-cycle pulses at $2 \mu \mathrm{m}$ wavelength. Opt. Lett. 40, 5160-5163 (2016).

37. Stutzki, F. et al. $152 \mathrm{~W}$ average power Tm-doped fiber CPA system. Opt. Lett. 39, 4671-4674 (2014). 